\title{
Posterior Hip Dislocation in Children: A Mild Traumatic Event. Case Report and Review of Literature
}

\author{
Van de Vyver A*, Van Assche A, Cuypers L and Jansegers E \\ Department of Orthopaedic Surgery and Traumatology, Belgium \\ *Corresponding author: Van de Vyver A, Department of Orthopaedic Surgery and Traumatology, Belgium

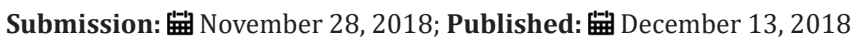

\begin{abstract}
Introduction: Traumatic posterior dislocation of the hip in children is an uncommon injury. Under 10 years of age it is often caused by a low-energy trauma such as typical child play. It requires urgent reduction to reduce chance of avascular necrosis.

Case report: A 9-year old boy was admitted after a fall from a trampoline. Clinical examination and radiographs revealed a left posterior hip dislocation without any associated fractures. Urgent closed reduction was performed within 6 hours resulting in a stable hip joint. Post-reduction radiographs and additional MRI showed a concentrically reduced hip. Skin traction was applied for 4 days and non-weightbearing suggested for 6 weeks.
\end{abstract}

Conclusion: Posterior traumatic hip dislocation in children is a rare urgency with favorable outcome if treated by quick reduction. Additional imaging is advised to guide adequate treatment.

Keywords: Pediatric traumatic hip dislocation; Imaging; Treatment; Avascular necrosis

\section{Introduction}

Traumatic dislocation of the hip in children is an uncommon injury, with a prevalence of $0.8 / 1.000 .000$ per year in children under 14 years. Of all traumatic hip dislocations, only $5 \%$ occur in children [1]. We report the case of a traumatic posterior hip dislocation in a 9-year old boy without any associated fractures.

\section{Case Report}

\section{History}

A healthy 9-year old boy was admitted to the emergency department after falling from a trampoline. During casual play our patient tripped and experienced heavy pain in the left leg afterwards. The pain did not seem to correlate with the energy of the fall. Weight bearing was impossible. He did not have any numbness in the left leg but noted a "bony" bump in his posterior thigh. When admitted at the hospital he had already received a great number of painkillers, including opiates.

\section{Clinical examination}

The left leg was internally rotated and adducted. There was slight flexion in the hip of about $20^{\circ}$. We noted normal sensation in the entire leg and full active dorsal- and plantar flexion.

\section{Imaging}

AP X-ray of the pelvis and hip demonstrated a posterior dislocation of the left hip without any associated fractures.

\section{Treatment}

A closed reduction (Allis maneuver), under general anesthesia, was performed in the operating theatre 4 hours after the event. Reduction was successful with a stable full range of motion. Postreduction X-ray and MRI showed no fractures or intra-articular pathology. Our patient was observed during 3 days with continuous skin traction of the left leg. 6 weeks non-weightbearing scheme was proposed.

\section{Discussion \\ Epidemiology}

Traumatic dislocations of the hip in children are uncommon. $80 \%$ of these are posterior dislocations caused by axial force on a flexed, adducted and internally rotated hip joint [2]. Due to ligament laxity and a soft pliable acetabulum this emergent injury can be caused by low energy traumas such as typical child play or even tripping without associated fractures [3]. In children older than 10 years, high energy (e.g. MVA) is needed to cause this injury and associated fractures are thus more common [4] (Figure 1)

\section{Diagnosis}

Low energy trauma and a leg in fixed internal rotation, adduction and slight flexion can guide to a correct diagnosis. AP pelvis- and hip radiographs confirm diagnosis and warrant for emergent closed reduction if no associated fractures are present. 


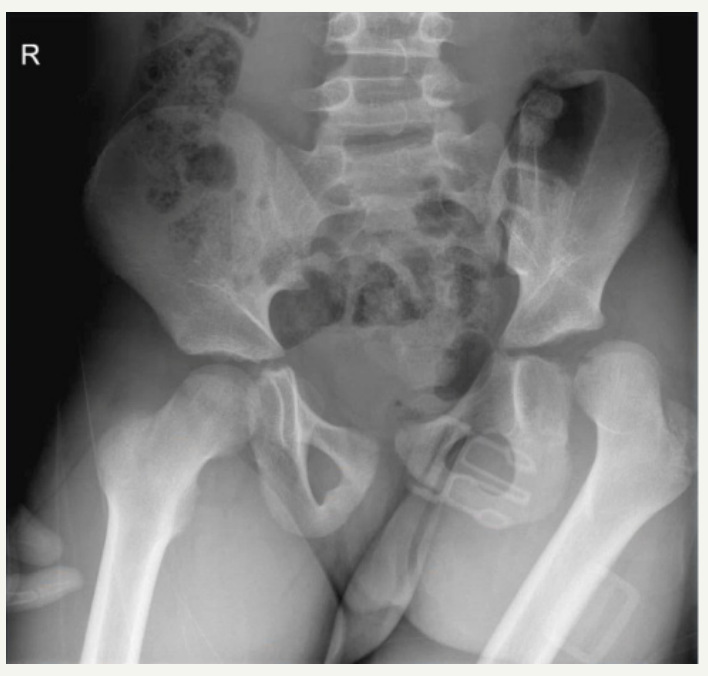

(a)

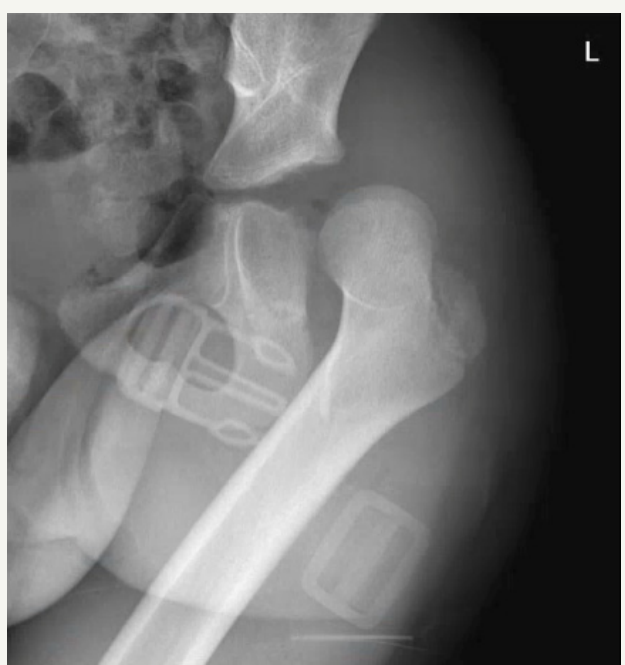

(b)

Figure 1: AP pelvis- and hip X-ray showing posterior hip dislocation without associated fractures.

\section{Acute treatment}

By pulling just below the knee, traction in line with the femur will reduce the hip joint if countertraction is applied by pushing both anterior superior iliac spines. Adduction and gentle rotation (both internal and external) will reduce the femoral head across the posterior acetabular wall [5]. This procedure is successful in

\section{Additional imaging}

most cases resulting in anatomic concentric reduction [6]. When associated fractures are present, open fracture fixation is needed before performing femoroacetabular reduction [5]. Possible causes for failed closed reduction in simple acute cases are intra-articular bone fragments, loose bodies or interposed soft tissue (posterior capsule, posterior chondrolabral complex detachment, piriformis muscle) [2].

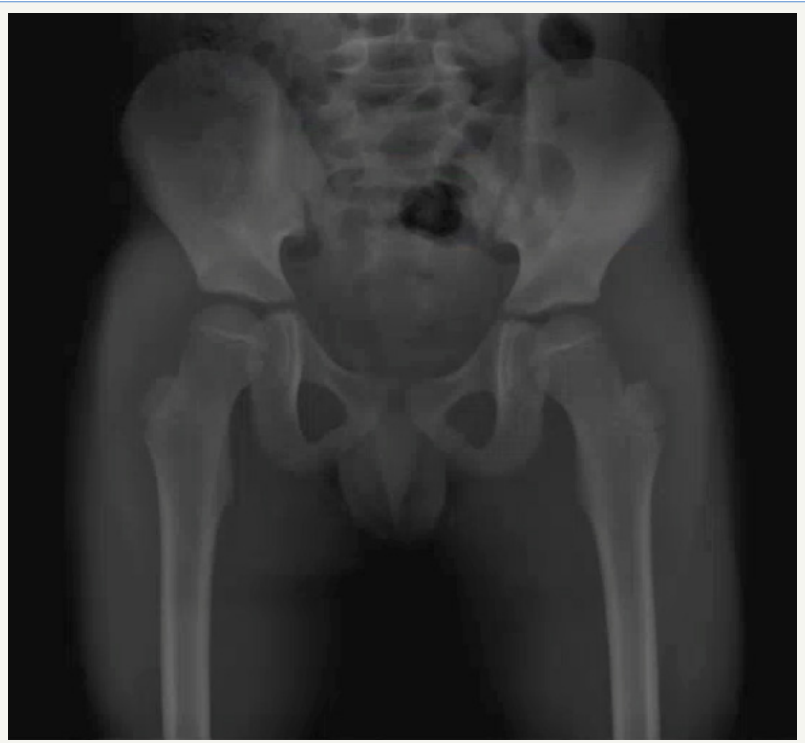

Figure 2: AP pelvis x-ray shows concentric anatomic reduction.

AP pelvis x-rays are needed after every closed reduction. Even in successful procedures, post-reduction x-rays may show asymmetrical hip joint widening compared to the other side.

Radiographic joint widening can be caused by intra-articular bone fragments (not always visible on x-ray), soft tissueinterposition such as mentioned above, or even simple hemarthrosis. Some studies suggest a routine drainage of hip hemarthrosis after every dislocation, though this is not generally supported [7]. Mehlman et al. [2] suggested computed tomography (CT) if hip joint asymmetry is $\geq 2 \mathrm{~mm}$ (measured from the medial edge of the femoral head ossification center to the lateral aspect of the acetabular tear figure). Other authors suggest post-reduction CT if there is gross hip instability after reduction or if ipsilateral periarticular fractures are present [8]. CT might show the typical acetabular fleck sign, suggestive of posterior acetabular osteochondral injury [9] (Figure 2 \& 3). 

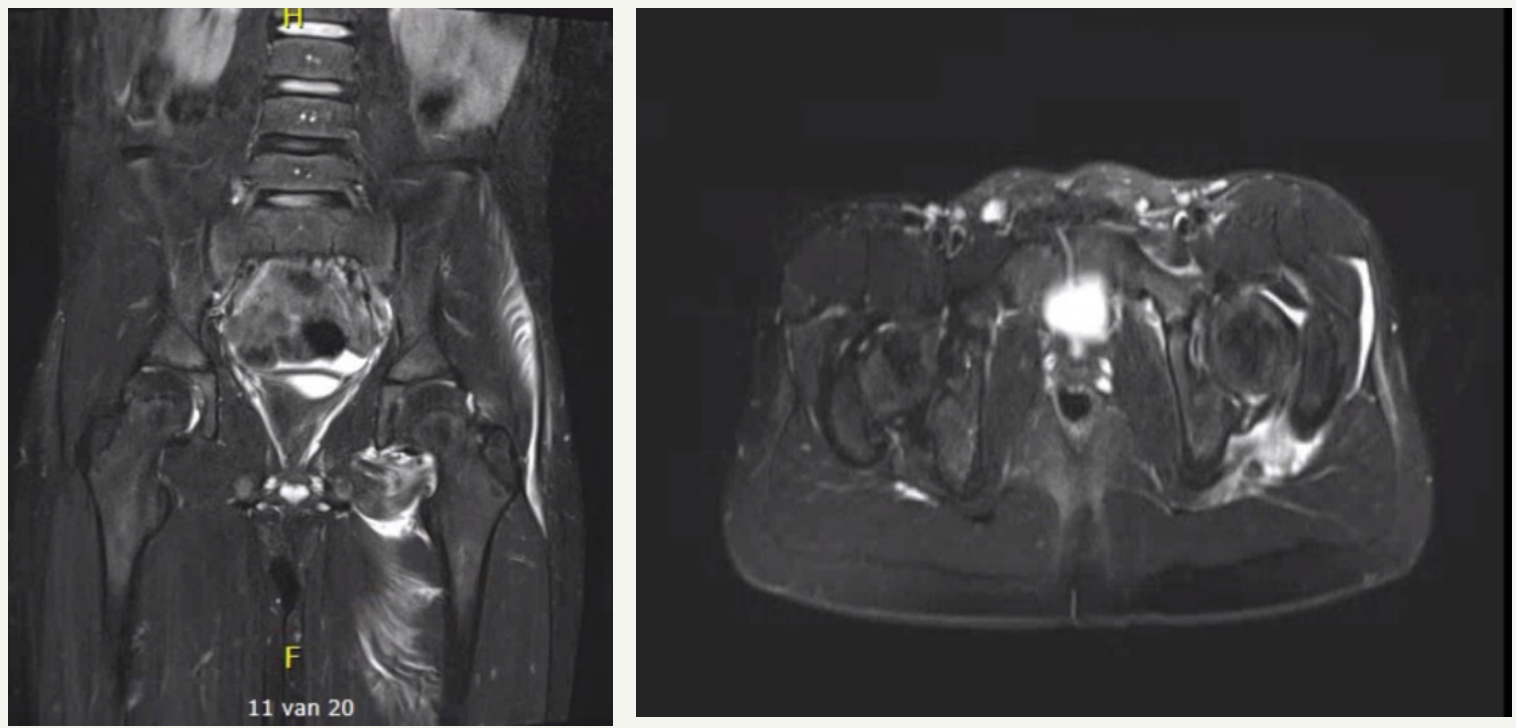

Figure 3: MRI after reduction shows IA fluid and adductor muscle contusion. No labral tears or loose bodies were visualized.

More recent studies favor the use of magnetic resonance imaging (MRI) $[10,11]$. There is no radiation exposure of the young child and soft tissue injuries can be easily diagnosed. Mayer et al. [11] performed a study comparing MRI and surgical findings. MRI detected all complications such as labral tears, ligamentum teres ruptures, bone marrow contusion, osteochondral intra-articular fragments, femoral head fractures and posterior acetabular wall fractures. Since posterior acetabular wall ossification is only complete at the age of 12 years in girls and 14 years in boys, CT underestimates these types of fractures. Size of the cartilaginous fragment was noted as length of the intact posterior wall compared to the other side $(30-70 \%$ in their cohort).

\section{Post-reduction treatment}

There is no consensus about post-reduction treatment. Both hip spica and skin traction have been suggested [1,2,5,12-14]. Mehlman et al. [2] applied a hip spica for 6 weeks in young patients (average 5 years) and skin traction for a short period in older patients (average 12 years). Afterwards protected weight bearing was suggested. This allowed for posterior soft tissues to heal. No recurrent dislocations were seen afterwards. Surgery is needed in cases of non-concentric reduction. There is much debate about open vs arthroscopic procedures. In open procedures, a posterior approach is favored with minimal distal extent to the proximal edge of quadratus femoris muscle. In most cases this is enough to reduce interposed soft tissue while not compromising vascularity. Recently, there has been a trend towards arthroscopic procedures to reduce interposition of soft tissue or remove loose bodies. There is no lower age limit for this procedure, though main limitation is obtaining safe adequate traction [6]. Bucket handle tears of the posterior labrum are generally reduced and repaired by an open posterior approach. One study showed spontaneous healing of a bucket-handle posterior labral detachment in a 5-year old child with a concentrically stable hip after 70 days [15]. Open surgical repair remains the gold standard for these lesions but future studies might focus on arthroscopic repair or even the spontaneous healing characteristics of capsulolabral tears [6].

In neglected cases where dislocation has persisted more than 3 weeks closed reduction is mostly impossible and open procedures should be advocated [16].

\section{Outcome}

Outcome after traumatic hip dislocations in children are usually satisfactory. Though rare, the most common complication is sciatic nerve injury (5-20\%) [16]. Avascular necrosis (AVN) of the femoral head $(3-15 \%)$ is associated with time interval to reduction. A reduction performed $>6$ hours after trauma shows a 20 -fold increased risk of AVN [2]. No significant relation with trauma impact, patient age or associated fractures was found. There is a higher risk of AVN in young boys since younger children have an incomplete anastomotic network between medial and lateral circumflex femoral arteries with absent transepiphyseal vessels. On top of that, boys seem to have less intracapsular anastomotic networks compared to girls $[17,18]$ A flow below $20 \%$ of normal is needed to initiate the process of AVN.

AVN becomes apparent on MRI between 2-12 months after dislocation. Bone scan is not advised since AVN can occur despite normal results (sensitivity 0\%, specificity 100\%) [2]. Coxa magna, defined as $\geq 2 \mathrm{~mm}$ difference in maximum femoral head diameter is a complication without clinical relevance. It is caused by reactive hyperemia of the femoral head [19]. Recurrent dislocations seem to be related to short periods of immobilization, not allowing for the gap between obturator internus and quadratus femoris to heal [1]. Other complications of posterior traumatic hip dislocation are heterotopic ossification, premature epiphyseal fusion and posttraumatic degenerative osteoarthritis caused by prolonged cartilage nourishment disturbances [13]. 


\section{Conclusion}

Posterior traumatic hip dislocation in children is a rare, emergent event with favorable outcome if treated appropriately. MRI is advised in cases of non-concentric reduction without risks of radiation exposure and good visualization of post-reduction hip anatomy.

\section{References}

1. Bressan S, Steiner IP, Shavit I (2014) Emergency department diagnosis and treatment of traumatic hip dislocations in children under the age of 7 years: a 10-year review. Emerg Med J 31(5): 425-431.

2. Mehlman CT, Hubbard GW, Crawford AH, Roy DR, Wall EJ (2000) Traumatic hip dislocation in children. Long-term followup of 42 patients. Clin Orthop Relat Res (376): 68-79.

3. Hamilton PR, Broughton NS (1998) Traumatic hip dislocation in childhood. J Pediatr Orthop 18(5): 691-694.

4. Barquet A (1979) Traumatic hip dislocation in childhood. A report of 26 cases and review of the literature. Acta Orthop Scand 50(5): 549-553.

5. Yuksel S, Albay C (2017) Early reduction of pediatric traumatic posterior hip dislocation is much more important than the treatment procedure. Pediatr Emerg Care doi: 10.1097/PEC.0000000000001351.

6. Morris AC, Yu JC, Gilbert SR (2017) Arthroscopic treatment of traumatic hip dislocations in children and adolescents: a preliminary study. J Pediatr Orthop 37(7): 435-439.

7. Rieger H, Pennig D, Klein W, Grünert J (1991) Traumatic dislocation of the hip in young children. Arch Orthop Trauma Surg 110(2): 114-117.

8. Frick SL, Sims SH (1995) Is computed tomography useful after simple posterior hip dislocation? J Orthop Trauma 9(5): 388-391.
9. Blanchard C (2016) Traumatic, posterior pediatric hip dislocations with associated posterior labrum osteochondral avulsion: recognizing the acetabular "fleck" sign. J Pediatr Orthop 36(6): 602-607.

10. Fabricant PD (2013) A radiographic study of the ossification of the posterior wall of the acetabulum: implications for the diagnosis of pediatric and adolescent hip disorders. J Bone Joint Surg Am 95(3): 230236.

11. Mayer SW (2015) MRI as a reliable and accurate method for assessment of posterior hip dislocation in children and adolescents without the risk of radiation exposure. Pediatr Radiol 45(9): 1355-1362.

12.Zrig M, Mnif H, Koubaa M, Abid A (2009) Traumatic hip dislocation in children. Acta Orthop Belg 75(3): 328-333.

13. Sulaiman AR, Munajat I, Mohd FE (2013) Outcome of traumatic hip dislocation in children. J Pediatr Orthop B 22(6): 557-562.

14. Madhu TS (2013) Treatment of developmental dislocation of hip: does changing the hip abduction angle in the hip spica affect the rate of avascular necrosis of the femoral head? J Pediatr Orthop B 22(3): 184188.

15. Clement RC, Carpenter DP, Cuomo AV (2018) Spontaneous healing of a bucket-handle posterior labral detachment after hip dislocation in a five-year-old child: a case report. JBJS Case Connect 8(2): e28.

16. Hung NN (2012) Traumatic hip dislocation in children. J Pediatr Orthop B 21(6): 542-551.

17. Chung SM (1976) The arterial supply of the developing proximal end of the human femur. J Bone Joint Surg Am 58(7): 961-970.

18. Yue JJ (2001) Blood flow changes to the femoral head after acetabular fracture or dislocation in the acute injury and perioperative periods. J Orthop Trauma 15(3): 170-176.

19. Offierski CM (1981) Traumatic dislocation of the hip in children. J Bone Joint Surg Br 63-B(2): 194-197.
Creative Commons Attribution 4.0 International License

For possible submissions Click Here

\section{Submit Article}

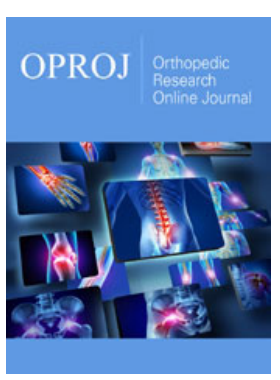

Orthopedic Research Online Journal

\section{Benefits of Publishing with us}

- High-level peer review and editorial services

- Freely accessible online immediately upon publication

- Authors retain the copyright to their work

- Licensing it under a Creative Commons license

- Visibility through different online platforms 